\title{
Discussion on the application of CCTV detection in the pipeline network inspection project of a community repair project in Shanghai
}

\author{
Fuyi \\ Shanghai Urban Construction Vocational College, Shanghai, China
}

\begin{abstract}
Taking the pipeline inspection as an example, this paper elaborates the CCTV detection process, and expounds the corresponding technical points combined with the construction site and relevant specifications. According to the inspection results, the defects of the pipeline are evaluated, and the corresponding maintenance and repair opinions are proposed.
\end{abstract}

\section{Introduction}

CCTV pipeline detection is a technical method to detect the deformation, rupture, collapse, blockage, poor drainage, leakage, direct and mixed drainage of rainwater and sewage within the scope of the project by using CCTV camera detector, and issue detection and evaluation report (including relevant field data and treatment opinions and suggestions).

The sewage intercepting pipe network has been built in a district of Shanghai for a long time and lacks management and maintenance. According to the statistics of the test results, it is found that there are structural defects such as rupture, disconnection, leakage and corrosion, and functional defects such as tree roots, obstacles, deposition and scaling, which have seriously affected the operation of the pipeline network. Combined with the actual situation of the site, priority should be given to solving structural defects above level 3 and all functional defects. The trenchless repair technology is used to repair the pipeline as a whole and as a local point. After repair, the pipeline returned to normal operation, which greatly improved the sewage interception efficiency of the area.

\section{CCTV detection technology}

\subsection{Application scope of CCTV detection technology}

Before CCTV pipeline detection, in order to clearly understand the actual situation inside the pipeline, the water level inside the pipeline needs to be detected is less than $20 \%$ of the pipe diameter. Before the implementation of detection, it is necessary to block, dredge and drain the pipeline, and then implement CCTV detection. Before the detection, the pipeline shall be carefully inspected, the well cover shall be opened,the flow direction and water color in the well shall be checked, and whether the pipe network is blocked and whether there is rain and sewage mixed connection; if the water level is more than $20 \%$, the pipeline shall be temporarily blocked for pumping, dredged and cleaned, and the water shall be used after meeting the detection requirements CCTV inspection, at the same time, refer to $\mathrm{QV}$, sonar and other detection means to determine whether the internal structure of the pipeline is damaged, whether there is concealed pipe access and the degree of silting. If some sewage pipelines cannot be detected by CCTV, other technical methods such as pipeline rapid detector shall be considered.

In case of any of the following situations, we will stop the detection of this section, and then carry out the test after solving the problem:

- Crawler cannot walk

- The lens is stained with water and mud, which affects the image quality

- Lens in water

- The pipe is full of fog

\subsection{CCTV detection technical process of the project}

\subsubsection{Structural testing}

Data collection - site survey - plugging and pumping dredging and cleaning - CCTV testing - Preparation of structural test report.

\subsubsection{Functional testing}

Data collection - site inspection - plugging pumping creeping walking - CCTV testing - Preparation of functional test report. 


\subsubsection{Specific steps of CCTV detection}

Firstly, the remote control color CCTV viewing lens is sent into the cleaned drainage pipeline, and the situation in the pipeline is transmitted to the TV monitoring screen and computer at the same time.

The operator's special recording can check the whole detection process one by one and record them into digital image files and store them in the hard disk of the computer. If there are abnormal phenomena in the detection process, the operator can operate the equipment to rotate and check the details of the defect points in detail. In order to facilitate the analysis of the inspection results in the later stage, the abnormal points will stay for a moment during CCTV detection to obtain clear images;

For pipeline leakage, pipe branch pipe concealed connection and other defects need to be recorded in detail, and the operation equipment should be carefully inspected.

According to the requirements of Shanghai relevant codes for the preparation of CCTV test report for drainage pipeline, the preparation of the drainage pipeline inspection report is completed.

After the completion of CCTV detection, all the files (image files) generated in the detection process will be converted into file formats that can be browsed and played on the client's computer, and engraved into CDs for users to use.

Due to the different aging degree of pipelines and the uncertainty of existing pipeline direction and location, some pipelines may not be included in the plan. Therefore, some sewage pipelines may be increased or decreased according to actual needs in the process of project development, so as to avoid the situation that important pipelines are missed. During the inspection process, the pipe diameter, buried depth, distance(Each road intersection and between the intersections is a unit of measurement, and it is indicated in the drawing) and underground well location of the project pipeline(Locate the exact location of the underground well in the pipeline and indicate in the drawing) shall be determined And provide the electronic network management diagram (CAD). Provide drawings to mark the well number of the inspected pipe section in the well chamber. The video data in the pipeline must be clear, continuous and complete, which can accurately reflect the internal situation of the pipeline, and the marked well number shall be photographed in the video.

\subsection{CCTV detection technical parameters of the project}

CCTV detection system is composed of main control display unit, pipeline crawling robot, cable reel and professional video image interpretation software. It has the comprehensive functions of camera, camera, editing, playback, storage, slope angle measurement, automatic forward and backward, turning, strong climbing ability and other comprehensive functions. It supports the function of automatically generating the test report under the specified standard according to the test results.

The pipeline network inspection system of X5 pipeline CCTV endoscope detection system is adopted for pipeline network inspection of the project repair project. The technical indexes are as follows: applicable pipe diameter: x5-s: $225 \mathrm{~mm}-2000 \mathrm{~mm}$, protection grade: IP68, x5-m: $150 \mathrm{~mm}-2000 \mathrm{~mm}$ rotating zoom lens / direct vision lens / fish eye lens / imaging chip: color $1 / 4$ "CCD resolution: $\geq 440000$ pixels, sensitivity: 0.1 lux Working temperature: $-10^{\circ} \mathrm{C}-50^{\circ} \mathrm{C}$

The system has the following technical characteristics and performance.

- Applicable pipe diameter: $\Phi 250-\Phi 1500$, waterproof performance: $10 \mathrm{M}$ water pressure.

-The length of the pipeline is measured to determine the specific location of the damage. The maximum length of a detection can reach $140 \mathrm{~m}$.

- The camera can rotate 360 degrees and squint \pm 120 degrees to take pictures in the tube, and can provide clear color images of the situation in the pipeline.

-It is light and compact with good performance, which ensures the smooth progress of the investigation.

\section{Test results and analysis}

According to the requirements of "technical specification for Trenchless repair and renewal engineering of urban drainage pipeline", the defects and abnormal conditions such as dislocation of original pipe joint, crushing and collapse of pipe wall were found out, and their position and defect grade were recorded in detail.

Through the preliminary investigation and drainage, the pipeline network in this area is finally tested. According to the CCTV inspection and evaluation report of the pipeline, the structural defects of the pipeline in this area are shown in the table 
Table1. The number and proportion of different types of structural defects.

\begin{tabular}{|c|c|c|c|c|c|}
\hline Defect name & $\begin{array}{c}\text { I } \\
\text { grade }\end{array}$ & $\begin{array}{c}2 \\
\text { grade }\end{array}$ & $\begin{array}{c}3 \\
\text { grade }\end{array}$ & $\begin{array}{c}4 \\
\text { grade }\end{array}$ & proportion \\
\hline fracture & $\mathbf{3 0 1}$ & $\mathbf{6 3 2}$ & $\mathbf{1 9 9}$ & $\mathbf{1 8 7}$ & $\mathbf{2 6 . 8 \%}$ \\
\hline deformation & $\mathbf{5 8}$ & $\mathbf{8 7}$ & $\mathbf{4 5}$ & - & $\mathbf{7 . 8 \%}$ \\
\hline dislocation & $\mathbf{5 5}$ & $\mathbf{1 1 2}$ & $\mathbf{7 4}$ & $\mathbf{2 3}$ & $\mathbf{1 0 . 9 \%}$ \\
\hline Disjointed & $\mathbf{2 3}$ & $\mathbf{2 2}$ & $\mathbf{5 0}$ & $\mathbf{3 4}$ & $\mathbf{5 . 3 \%}$ \\
\hline leakage & $\mathbf{3 8 2}$ & $\mathbf{4 3 2}$ & $\mathbf{2 3 1}$ & $\mathbf{2 9}$ & $\mathbf{4 4 . 2 \%}$ \\
\hline $\begin{array}{c}\text { Pipeline } \\
\text { invasion }\end{array}$ & $\mathbf{3 9}$ & $\mathbf{5 1}$ & $\mathbf{3 0}$ & - & $\mathbf{4 . 9 4 \%}$ \\
\hline
\end{tabular}

Table2. The number and proportion of different types of functional defects.

\begin{tabular}{|c|c|c|c|c|}
\hline Defect name & $\begin{array}{c}\text { I } \\
\text { grade }\end{array}$ & $\begin{array}{c}2 \\
\text { grade }\end{array}$ & $\begin{array}{c}3 \\
\text { grade }\end{array}$ & proportion \\
\hline deposition & $\mathbf{8 0}$ & $\mathbf{1 3 4}$ & $\mathbf{5 6}$ & $\mathbf{2 7 . 9 \%}$ \\
\hline Scaling & $\mathbf{5 1}$ & $\mathbf{8 3}$ & $\mathbf{2 5}$ & $\mathbf{1 2 . 3 \%}$ \\
\hline obstacle & $\mathbf{9 2}$ & $\mathbf{1 0 3}$ & $\mathbf{4 7}$ & $\mathbf{2 0 . 5 \%}$ \\
\hline tree root & $\mathbf{1 2 3}$ & $\mathbf{2 3 9}$ & $\mathbf{4 5}$ & $\mathbf{3 4 . 1 \%}$ \\
\hline $\begin{array}{c}\text { Depression } \\
\text { water }\end{array}$ & $\mathbf{8}$ & $\mathbf{3}$ & $\mathbf{1}$ & $\mathbf{3 . 2 \%}$ \\
\hline Dam head & $\mathbf{6}$ & $\mathbf{5}$ & $\mathbf{3}$ & $\mathbf{1 . 1 \%}$ \\
\hline dross & $\mathbf{5}$ & $\mathbf{4}$ & $\mathbf{2}$ & $\mathbf{0 . 8 \%}$ \\
\hline
\end{tabular}

The pipeline diseases in different regions are different. In order to formulate the corresponding maintenance and repair scheme of the pipeline, it is necessary to detect the pipeline. Through the detection, the location and severity of the pipeline problems are found, so as to provide reference for the formulation of the scheme.

At present, among many detection methods, CCTV detection is the most intuitive detection method, and its construction is convenient and fast.

\section{References}

1. Saeed Moradi; Tarek Zayed;Fuzhan Nasiri; Farzaneh Golkhoo Journal of Infrastructure Systems 2020
2. Srinath Shiv Kumar; Mingzhu Wang; Dulcy M. Abraham; Mohammad R. Jahanshahi; Tom Iseley Deep Learning-Based Automated Detection of Sewer Defects in CCTV Videos Journal of Computing in Civil Engineering 2020

3. Hubert Cecotti, Agustin Rivera, Majid Farhadloo et al. Grape detection with convolutional neural networks[J] Expert Systems With Applications, 2020, 159

4. Application of Pipeline Detection Technology in the Reconstruction of the City Roads;zhangchao; Geomatics Science and Technology; 2020, 8(02):5459

5. Zhejiang Future Technology Institute (jiaxing); Patent Application Titled "Methods And Systems For 
Pipe Wall Thickness Detection" Published Online (USPTO 20200300812). 2020, :5657-.

6. Tara Moghareh Abed, Mehran Eskandari Torbaghan, Aryan Hojjati, et al. Experimental Investigation into the Effects of Cast-Iron Pipe Corrosion on GPR Detection Performance in Clay Soils; Journal of Pipeline Systems Engineering and Practice2020, 11(4) 